\title{
AFLPs do not support deep phylogenetic relationships among darters (Teleostei: Percidae: Etheostomatinae)
}

\author{
Heredity (2012) 108, 647-648; doi:10.1038/hdy.2011.130; published online 11 January 2012
}

In a recent paper Smith et al. (2011) presented an amplified fragment length polymorphism (AFLP) data set from 20 primer pairs that were scored for 69 darter species to infer phylogenetic relationships. Our primary concern with the conclusions presented in Smith et al. (2011) is an overstatement of the phylogenetic utility of ALFP markers for deep relationships among darters, and support for taxonomic groups inferred from non-phylogenetic assessments of morphology. Despite claims to the contrary, the Bayesian phylogeny presented in Figure 2 of Smith et al. (2011) conclusively demonstrates that AFLP markers do not provide strong support for deep relationships among darters, nor does phylogenetic analysis of this data support a majority of the sampled taxonomic groups. In addition, we present a new analysis that shows AFLP markers have a very low probability of providing strong posterior clade support $(\geqslant 0.95)$ in Bayesian analysis for the nodes representing the oldest lineages in the darter radiation, whereas a recently published analysis of a modest DNA sequence data set provides substantially greater support for these relationships (Near et al., 2011).

It is a fact that all of the deep clades in the AFLP-inferred phylogeny, with the exception of the most recent common ancestor of all darters, are poorly supported with Bayesian posterior probabilities that are all $<0.90$, directly contradicting the claims by Smith et al. (2011) in the title of their paper, which promises phylogenetic support for deep relationships among darters. Smith et al. (2011) also claim that their analyses of AFLP characters support groups that are consistent with morphological hypotheses. It is important to note that these hypotheses of darter taxonomic groups were not derived from explicit phylogenetic analyses, but are rather assumptions of relationships based on an assessment of the gestalt of an organisms overall appearance (Page 1974). In Table 1 of their paper, Smith et al. (2011) presented 23 polytypic darter taxonomic groups that were sampled with two or more species. Of these 23 groups, only 12 are resolved as monophyletic in the Bayesianinferred phylogeny presented in their Figure 2. It appears that ALFPs neither provide support for inferred relationships among the deepest nodes in the darter phylogeny, nor do they resolve an appreciable number of traditional darter taxonomic groups as monophyletic.

In Bayesian phylogenetic analyses, the posterior distribution of trees and parameter values provide an objective way to assess support for phylogenetic inferences (Huelsenbeck et al., 2001). The proportion of trees that include the clade is known as the posterior clade probability, which translates to the probability that the group is monophyletic, given the model and the data (Larget and Simon, 1999; Huelsenbeck and Rannala, 2004). Simulations have demonstrated that the posterior probability of a phylogeny is equal to the frequentist probability that the tree is correct when all assumptions of the Bayesian analysis are satisfied (Huelsenbeck and Rannala, 2004). This correlation has contributed to the common practice in Bayesian phylogenetics that a given node in a phylogeny is considered strongly supported if it is present in $\geqslant 95 \%$ in the posterior distribution of trees. Therefore, a clade that is not present in $\geqslant 95 \%$ of the posterior set of trees is not considered strongly supported in Bayesian phylogenetical analyses (Huelsenbeck et al., 2000).

The deep relationships that concern Smith et al. (2011) are focused on the monophyly of Etheostoma, as delimited in Page (2000), and the authors claim to support in their analyses of the AFLP data. We analyzed the AFLP data set (available at Heredity website) using MrBayes 3.0 (Ronquist and Huelsenbeck, 2003) and the exact settings as in Smith et al. (2011). Our analyses resulted in a very similar set of posterior trees, but our examination of this set of trees revealed that monophyly of Etheostoma was present in only $46 \%$ of the posterior distribution of trees. A Bayesian posterior probability of 0.46 is not typically offered as evidence for support of a clade, and few researchers would accept a hypothesis with a probability of 0.46 as correct.

There were other interesting relationships among the major darter lineages in the posterior phylogenies resulting from the Bayesian analysis of the AFLP data set not discussed by Smith et al. (2011). For example, $17 \%$ of the of the posterior distribution of trees contained a clade consisting of Carnipellucida (Ammocrypta + Crystallaria), Percina and Etheostoma cinereum; 14\% contained the clade listed above with the addition of Nothonotus; 7\% contained a clade composed of Carnipellucida and E. cinereum and $8.8 \%$ of the set of posterior trees resolved Etheostoma cinereum as the sister lineage of all other darters. The frequency of several alternative topologies in the posterior set of trees clearly showed the extensive phylogenetic uncertainty resulting from Bayesian analysis of the ALFP data set, which beguiles any attempt to characterize these deep relationships as supported.

We were interested in assessing the evolutionary age at which there is an expectation for strong posterior clade support in Bayesian analysis of AFLPs versus that of a recently published DNA sequence data set. Nodes in the Bayesian phylogeny presented in Figure 2 of Smith et al. (2011) were matched to 41 nodes containing the same subset of species in a time-calibrated phylogeny based on a multi-locus DNA sequence data set that sampled $98.8 \%$ of all darter species (Near et al., 2011). The matching nodes in the AFLP and DNA sequence-inferred phylogenies were each scored dichotomously for the presence (1) or absence (0) of strong posterior clade support $(\geqslant 0.95)$, and the estimated age of these nodes in the time-calibrated DNA-inferred phylogeny was recorded as a 


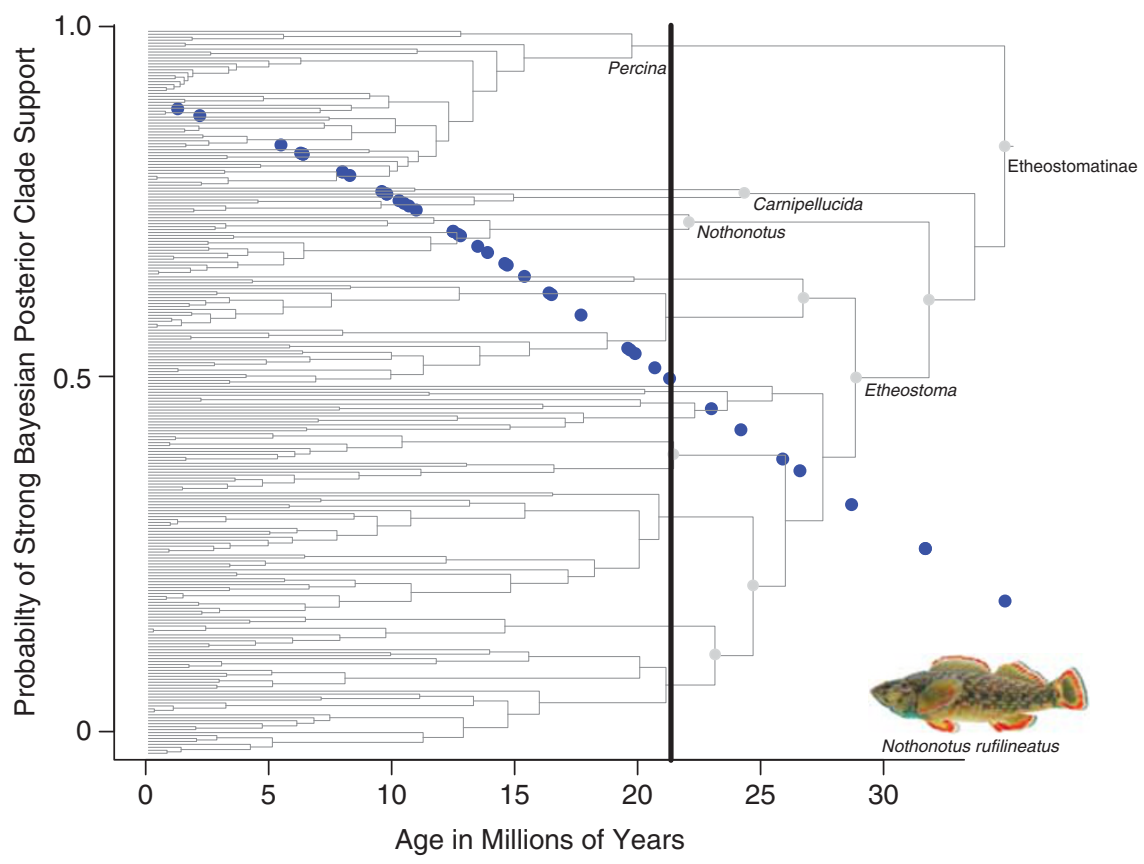

Figure 1 Logistic regression of probability for strong Bayesian posterior support ( $\geqslant 0.95)$ for nodes in the darter ALFP phylogeny (Smith et al., 2011) treated as a dichotomous variable and estimated age of the node in a relaxed molecular clock analysis (Near et al., 2011). Filled dark circles represent the logistic curve. The intercept was $21.3 \mathrm{Ma}$ and is marked with a black vertical line. Contrasts in the darter phylogeny that are older than the intercept are not expected to have strong posterior support in Bayesian phylogenetic analyses of AFLP data sets. Overlaid on the plot is a time-calibrated phylogeny inferred from a multi-locus data set scaled to the estimated evolutionary age. Nodes in the DNA sequence-inferred time tree that were $>21.3$ Ma and supported with Bayesian posteriors $\geqslant 0.95$ are marked with a filled grey circle. The most recent common ancestors of major darter clades resolved in the multi-locus time tree, which comprise the deepest relationships, are labelled.

continuous variable. We used two separate logistic regression analyses to determine the intercept of the continuous variable, estimated lineage age, where there was a $50 \%$ probability of observing a strong Bayesian posterior clade support for the node in the AFLP and DNA sequence-inferred phylogenies. The relationship between strong posterior Bayesian clade support and age of the node was negative and significant $(P<0.05)$ for the ALFP phylogeny. The relationships between strong posterior Bayesian clade support and age of the node was negative but not significant $(P=0.30)$ for the DNA phylogeny because nearly all of the sampled nodes were supported with strong Bayesian posterior probabilities. The intercept in the AFLP analysis was 21.3 million years (Ma), which indicates that lineages older than this age will have a greater probability of not having strong Bayesian support using AFLP data (Figure 1). The intercept in the DNA phylogeny was 53.0 Ma, which is an age that is at least $>20 \mathrm{Ma}$ than the estimated age for the most recent common ancestor of all darters (Near et al., 2011). Overlaying the time-calibrated phylogeny inferred from DNA sequence data onto the logistic regression plot of the ALFP Bayesian support versus age revealed that all of the reconstructed relationships among major darter lineages are much older than the estimated limit for AFLP support at $21.3 \mathrm{Ma}$. A total of 9 out of the 15 nodes that were older than the logistic regression intercept were supported with strong posterior values in the DNA-inferred phylogeny, as compared with only one in the AFLP phylogeny (Figure 1). It is clear that AFLPs do not resolve deep relationships among darters unless one rejects the practicing definitions of support in Bayesian phylogenetics.

\section{CONFLICT OF INTEREST}

The authors declare no conflict of interest.

TJ Near ${ }^{1}$ and BP Keck ${ }^{2}$ ${ }^{1}$ Department of Ecology \& Evolutionary Biology and Peabody Museum of Natural History, Yale University, New Haven, CT, USA and ${ }^{2}$ Department of Ecology and Evolutionary Biology, University of Tennessee, Knoxville, TN, USA E-mail: thomas.near@yale.edu

Huelsenbeck JP, Rannala B (2004). Frequentist properties of Bayesian posterior probabilities of phylogenetic trees under simple and complex substitution models. Syst Bio/ 53: 904-913.

Huelsenbeck JP, Rannala R, Masly JP (2000). Accommodating phylogenetic uncertainty in evolutionary studies. Science 288: 2349-2350.

Huelsenbeck JP, Ronquist F, Nielsen R, Bollback JP (2001). Bayesian inference of phylogeny and its impact on evolutionary biology. Science 294: 2310-2314.

Larget B, Simon DL (1999). Markov chain Monte Carlo algorithms for the Bayesian analysis of phylogenetic trees. Mol Biol Evol 16: 750-759.

Near TJ, Bossu CM, Bradburd GS, Carlson RL, Harrington RC, Hollingsworth Jr PR et al. (2011). Phylogeny and temporal diversification of darters (Percidae: Etheostomatinae). Syst Biol 60: 565-595.

Page LM (1974). The subgenera of Percina. Copeia 1974: 66-86.

Page LM (2000). Etheostomatinae. In: JF Craig (ed.). Percid Fishes: Systematics, Ecology and Exploitation. Blackwell Science: Oxford, pp 225-253.

Ronquist F, Huelsenbeck JP (2003). MrBayes 3: Bayesian phylogenetic inference under mixed models. Bioinformatics 19: 1572-1574.

Smith TA, Mendelson TC, Page LM (2011). AFLPs support deep relationships among darters (Percidae: Etheostomatinae) consistent with morphological hypotheses. Heredity 107: 579-588. 